\title{
Understanding backgrounds towards a first measurement of the top quark pair cross-section at ATLAS
}

\author{
Balint Radics*† \\ University of Bonn \\ E-mail: radicsephysik.uni-bonn.de
}

\begin{abstract}
Top-quark pairs are expected to be produced copiously at the LHC, even at the lower beam energy and luminosity expected in the first years of running. Establishing the top-pair signal and measuring the production cross-section are important benchmarks for ATLAS, and will help understand the detector performance for events with high-pT leptons, high jet multiplicity, missing transverse energy and b-jets. The prospects for these early top physics measurements will be shown, with a particular emphasis on the progress achieved with data so far.
\end{abstract}

The Xth Nicola Cabibbo International Conference on Heavy Quarks and Leptons, October 11-15, 2010

Frascati (Rome) Italy

\footnotetext{
*Speaker.

$\dagger$ on behalf of the ATLAS collaboration
} 


\section{Introduction}

The ATLAS experiment [1] has started to operate in early April 2010 along with the turn on of the Large Hadron Collider (LHC). Since then a successful operation has started and significant amount of $7 \mathrm{TeV}$ center-of-mass proton-proton collision data has been collected. The amount of data allows to start studies already on the performance of the ATLAS detector and to validate physics object reconstruction algorithms. With an integrated luminosity of $0.3 \mathrm{pb}^{-1}$ physics analyses have been started to establish the existence of benchmark processes such as top quark pair production. The main program of the LHC is to explore the energy scale beyond the Standard Model of particle physics for which these benchmark processes are essential ingredients. In the following the performance of the top quark pair production search of ATLAS is presented from the early data.

\section{The ATLAS detector}

The ATLAS (A Toroidal Lhc ApparatuS) detector is a multi-purpose particle detector and has been built around one of the bunch crossing collision points of the LHC. An overview of the ATLAS detector system is shown in Fig 1. The overall structure of ATLAS is driven by the eightfold azimuthal symmetry of its three large superconducting toroids (one barrel and two end-caps) which is completed by a superconducting solenoid. Going from inside out there is an inner detector providing pattern recognition, vertex finding and momentum measurements. It is made of silicon pixels and strips, and a straw-tube tracking detector capable of generating and detecting transition radiation. The inner detector sits in a $2 \mathrm{~T}$ solenoidal field. The solenoid is surrounded by a high granularity liquid-argon (LAr) electromagnetic calorimeter and the hadronic calorimeter which in the barrel region uses scintillator-tile detectors while in the forward region uses LAr technology. The muon spectrometer is the outermost detector built from tracking chambers.

\section{Strategy for top quark search}

In the early data the focus of top searches at ATLAS is on the single lepton plus jets final state of top quark pair production, $t+\bar{t} \rightarrow b \bar{b} W^{+} W^{-} \rightarrow b \bar{b} q \bar{q}^{\prime} l \bar{v}_{l}$, where the lepton, $l$, is an electron or a muon. ATLAS expects around 15 single lepton plus jets events to be produced in an integrated luminosity of $0.3 \mathrm{pb}^{-1}$. The main backgrounds for this signal are $W \rightarrow l \bar{v}+$ jets and QCD multijet production. The main event selection strategy is driven by the event topology of the single lepton plus jets event: in the analysis events are selected only if the ATLAS trigger system fires on a single lepton with large transverse momentum, then events are required to contain exactly one corresponding offline reconstructed isolated lepton, and furthermore the events are required to contain a significant amount of missing transverse energy due to the neutrino, both leptons expected to come from the decay of the massive $W^{ \pm}$vector boson. From the decay of the two top quarks to two bottom quarks and from the decay of the other $W^{ \pm}$vector boson to light quarks one expects the presence of at least four jets therefore events are selected where there is presence of jets with large transverse momentum. Additionally jets originating from $b$ quarks necessitates the usage of tagging algorithms to improve the top signal selection. 


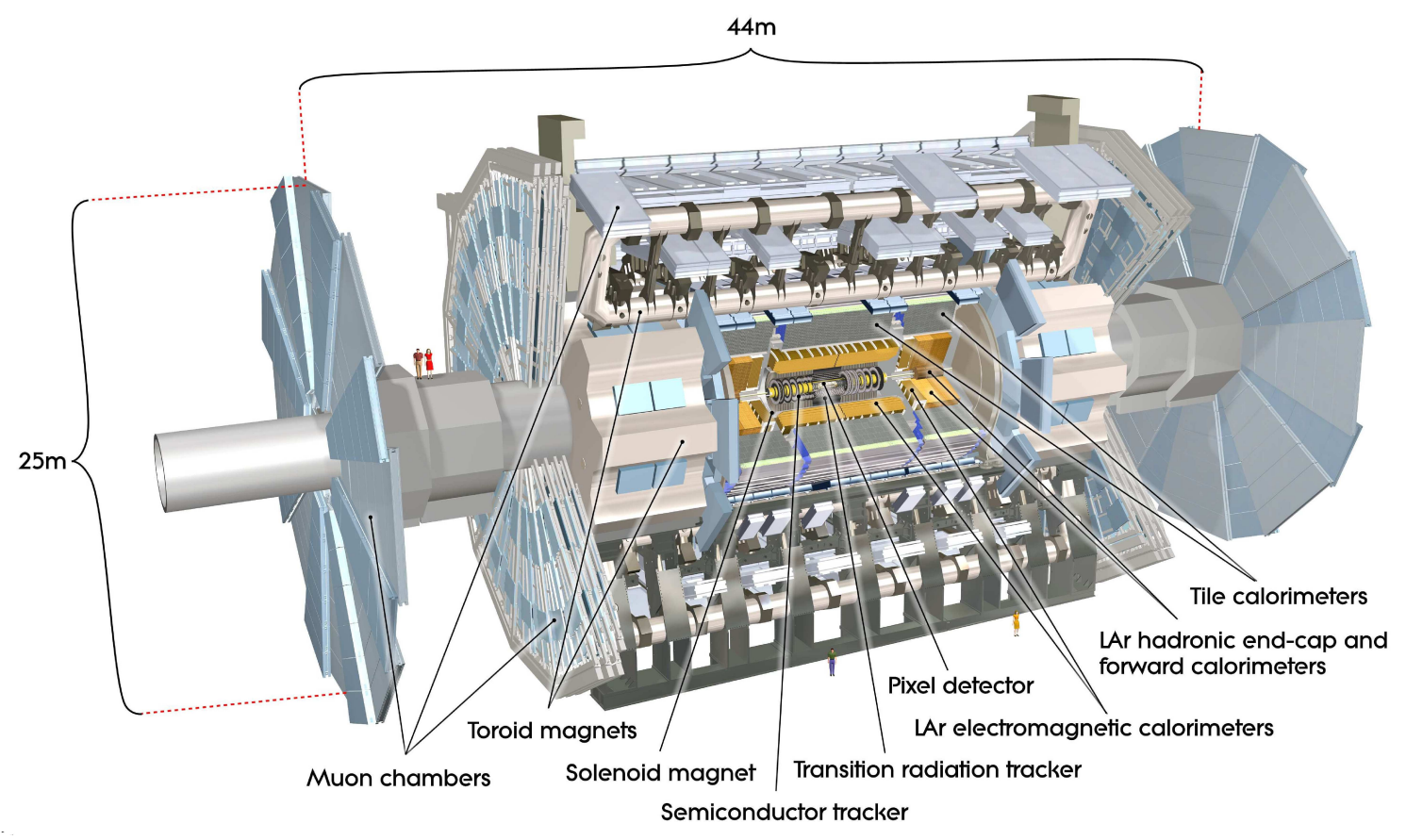

Figure 1: The ATLAS detector.

\section{Performance of reconstruction of physics objects}

In order to correctly select top quark pair candidate events the performance of electron, muon, jet, missing transverse energy and $b$-tagging has been validated.

Electron candidates [2] are reconstructed by associating inner detector tracks to clusters of locally deposited energy in the electromagnetic calorimeter. the At ATLAS the electrons are classified to medium and tight electrons using the various discriminators against other backgrounds. For medium electrons the high granularity of the first layer of the electromagnetic calorimeter is used to discriminate against pions by exploiting the electromagnetic shower shape and fractional energy distribution, as well as the ratio of the transverse energy deposited to the first layer of the hadronic calorimeter with respect to that of to the total electromagnetic calorimeter and the number of hits in the pixel detector. Tight electrons are defined as those electron candidates which have more than one hit in the inner-most (vertexing) layer of the pixel detector, and for the discrimination of these electrons the fraction of high threshold hits in the Transition Radiation Detector is also used. Comparisons of the distributions of these discriminators in data with the Monte Carlo simulations show a good agreement.

Muon candidates [3] are reconstructed from the combination of inner detector tracks with tracks in the muon spectrometer. Various distributions have been compared between data and Monte Carlo simulations, such as the number of Monitor Drift Tube hits, and good agreement has been found in the early data. The main tool for muon quality is the muon isolation. Muons originating from the hadronic decays within jet are usually in a more radiative environment than isolated muons from massive vector boson decays. This isolation is defined by forming a cone around the muon and 
summing up either the calorimeter energy or the momentum of the tracks - except for the muon candidate in question. This discriminator have also shown very good agreement between data and minimum bias Monte Carlo simulations.

Jets [4] [5] are reconstructed from calorimeter cells grouped into cluster. The main reconstruction algorithm used is the anti $-\mathrm{k}_{\mathrm{T}}$ with a parameter $R=0.4$ or 0.6 . Jets at this level are said to be calibrated to the electromagnetic scale because the energy depositions in the calorimeter clusters used for reconstruction are assumed to originate mainly from electromagnetic processes. Various schemes have been developed at ATLAS to correctly calibrate the jets from this electromagnetic scale to a hadronic scale. They either use Monte Carlo simulation information of simulated particle jets before detector simulation, or make use of the different response of the ATLAS calorimeters to hadronic and electromagnetic signals, or use individually calibration of clusters derived from Monte Carlo simulation of pions. In general rather good agreement have been found between jets reconstructed in the data and in the simulation in terms of calibration scale factors for all schemes. The main uncertainties are assigned by measuring the differences of these various calibration schemes. Jet energy scale uncertainty have been estimated at ATLAS using Monte Carlo simulations and resulted in an average value of 9-10\%, having dominant contributions from variations in the hadronic shower model and the amount of dead material in the detector.

The missing transverse energy [6] is calculated by taking the vector sum of the contributions from calorimeter cells of clusters above a certain pulse threshold, and adding muon contribution separately. The calibration of the missing transverse energy is done also using the procedure outlined for jets, and additional corrections are applied from the fully identified and offline reconstructed physics objects (electron, photon, muon, tau candidates and jets) and also using calorimeter cells not having been assigned to any of the reconstructed objects. The resolution of the missing transverse energy in the data have been compared with minimum bias Monte Carlo simulations and satisfying agreement has been found in the shape of the resolution function: $\sigma\left(\mathrm{E}_{\mathrm{x}, \mathrm{y}}^{\mathrm{miss}}\right)=0.5 \cdot \sqrt{\sum \mathrm{E}_{\mathrm{T}}}$. To select jets originating from $b$-quarks the so-called SV0 algorithm is used [7]. This algorithm uses the signed decay length significance, $\mathrm{L} / \sigma(\mathrm{L})$, as a main discriminator between light, charm and bottom quark jets. The tagger algorithm searches for displaced vertices with tracks having 2-track 3 dimensional impact parameter significance greater than 6. Also two-track resonances or tracks from photon-conversion are removed from consideration of the tracks of a vertex.The distribution of $\mathrm{L} / \sigma(\mathrm{L})$ has been compared between data and Monte Carlo simulations and a reasonable agreement has been found as well.

\section{Top quark search results}

For top quark pair search in the single lepton plus jets channel the event selection outlined in Section 3 has been used. After event selection the requirement of isolated single leptons suffer from QCD multijet background faking leptons originally expected to be coming from massive vector boson decay. The estimation of the multijet background is done by loosening and tightening the lepton isolation requirement for the events selected in the data, and assuming that the relative reduction of fake leptons due to the variation of the isolation is roughly constant and stable throughout the phase space of the events. This has been confirmed, from Monte Carlo simulations, to be true with an uncertainty of $\sim 20-30 \%$. The method is data-driven since it uses the data to 
establish the fake lepton response due to isolation variation.

Finally the following event selection has been applied on the data events [8][9]: only events were selected in which the primary vertex has at least 5 associated tracks, single lepton trigger fired for single lepton with at least $10 \mathrm{GeV}$ transverse momentum, the events must have exactly one offline reconstructed isolated lepton with a transverse momentum of at least $20 \mathrm{GeV}$, at least $20 \mathrm{GeV}$ missing transverse energy, and jets with at least $20 \mathrm{GeV}$ transverse momentum. The distributions of the reconstructed missing transverse energy and of the transverse mass of the $W$ boson (reconstructed from the lepton and the missing transverse energy) for electron and muon final states are shown on figure Fig 2.

The distributions show a remarkable good agreement between data and the Monte Carlo simulations within the statistical uncertainties in the data. The events are dominated by $\mathrm{W}+\mathrm{jets}$ and QCD multijet background. The shapes are very well estimated with the data-driven QCD multijet background estimation, the uncertainty of which is shown also as shaded area on top of the Monte Carlo simulations. The $\mathrm{W}+$ jets background produces a peak at the $\mathrm{W}$ boson mass of $80 \mathrm{GeV}$. The jet multiplicity distribution suggests the presence of top quark pair production in the inclusive four jet bin. Applying the SV0 b-tagging algorithm by requiring that there is at least one $b$-tagged jet in the events reduces the two major backgrounds and enhances the events with top quark pair events, as shown on Fig 3. After applying the $b$-tagging discriminator Monte Carlo simulation shows still a good agreement with the data withing the rather larger uncertainties due to both the statistical uncertainty of the data and uncertainty of the QCD background estimation.

\section{Summary}

The ATLAS experiment has been collecting data since April of 2010. The amount of data collected after only a few months of operation already allows to test the performance of physics object and particle reconstructions. Given the good agreement in the performance between Monte Carlo simulations and the data studies for search of top quark pair production have been started with an integrated luminosity of $0.3 \mathrm{pb}^{-1}$ of $7 \mathrm{TeV}$ proton-proton collision data. The main focus in the early data is on the single lepton plus jet final state of the top quark pairs. After event selection the data shows clearly the production of $\mathrm{W}$ bosons with associated jets and the estimation of the QCD multijet background together with a $b$-tagging algorithm shows already agreement in the Monte Carlo simulations compared with the data when top quark production is assumed as well. The final and clean observation of top quark pair production is to come after the full analysis of the data, collected in 2010 at ATLAS, has finished.

\section{References}

[1] G.Aad et al. (ATLAS), J.Inst. Vol. 3, August 2008

[2] ATLAS-CONF-2010-073

[3] ATLAS-CONF-2010-036

[4] ATLAS-CONF-2010-053

[5] ATLAS-CONF-2010-056 
[6] ATLAS-CONF-2010-057

[7] ATLAS-CONF-2010-042

[8] ATLAS-CONF-2010-063

[9] ATLAS-CONF-2010-087
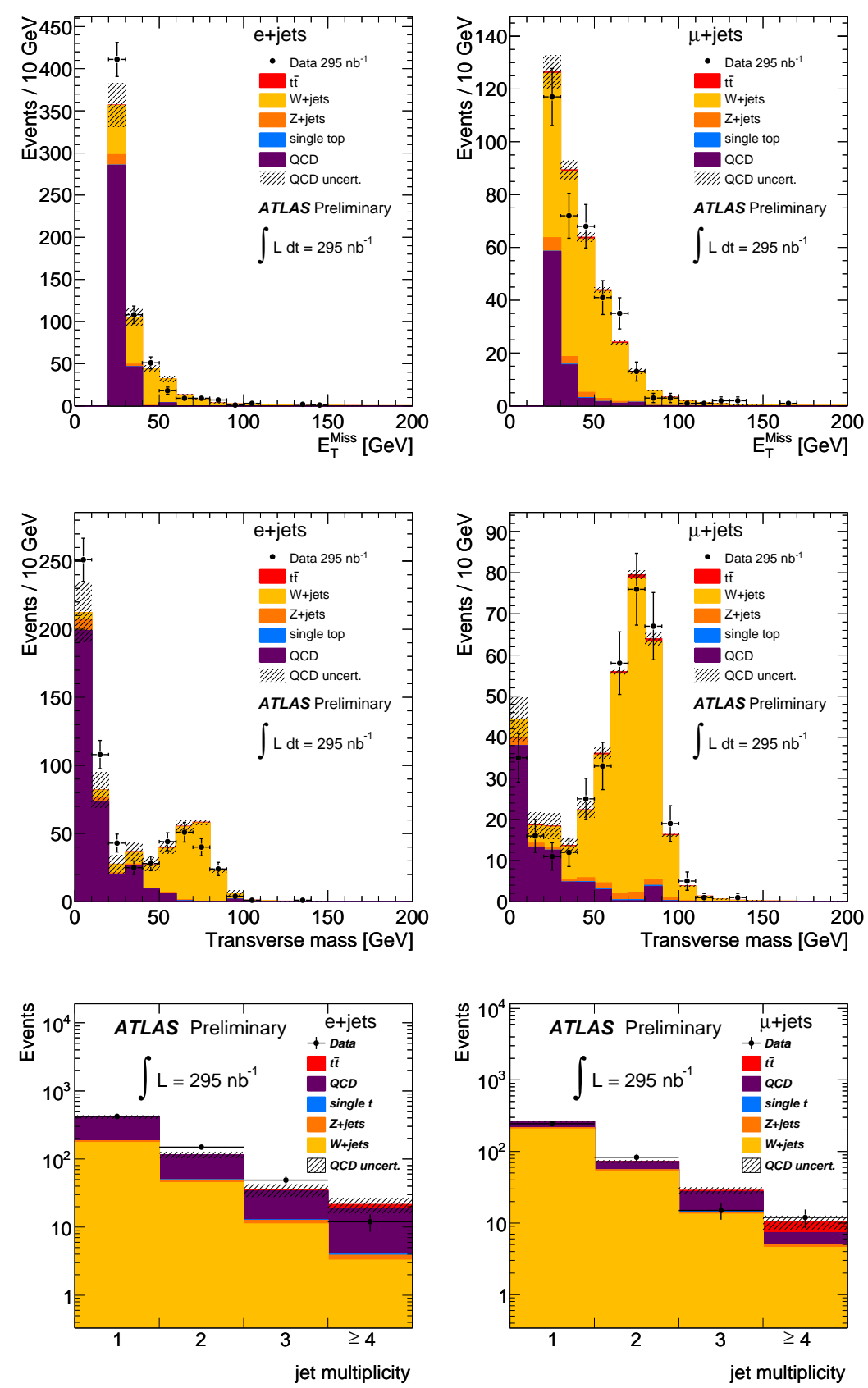

Figure 2: From top to bottom: distributions of missing transverse energy, reconstructed $W$ transverse mas and jet multiplicity after the event selection for single lepton plus jet channel (discussed in the text), lepton is either electron or muon. 

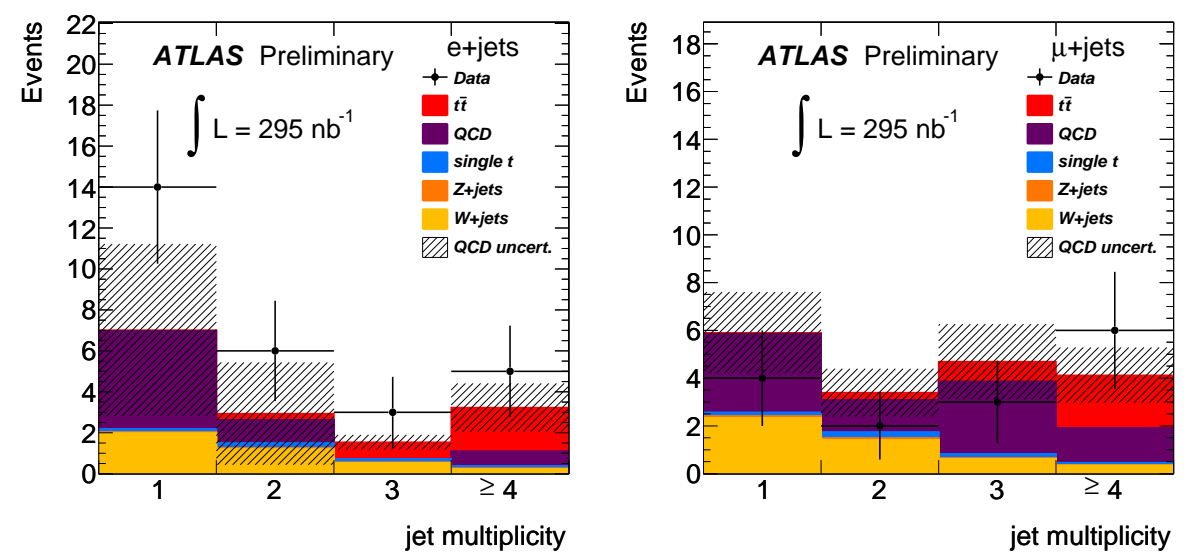

Figure 3: Distribution of jet multiplicity after the event selection for single lepton plus jet channel (discussed in the text), with requiring the presence of at least one b-tagged jet, lepton is either electron or muon. 